\section{Efficient Cloning of DAF Polymorphic Markers from Silver-Stained Polyacrylamide Gels}

BioTechniques 24:593-595 (April 1998)

DNA amplification fingerprinting (DAF) (1) gives about 40-60 bands per polyacrylamide gel lane, which makes the polymerase chain reaction (PCR) pattern rather complex for band isolation from a gel. The presence of minor amplified bands having only a few base pairs difference from major bands interferes with the product cloning because of co-isolation (3). This fact becomes obvious if a multiply re-amplified band is to be cloned. For cloning of a soybean polymorphic DAF marker (Figure 1A), we used the standard cloning procedure, in which the candidate DAF band is re-amplified 3-5 times to enrich the amount of PCR product (6). Instead of the expected unique 450-bp marker, we repeatedly obtained a set of cloned inserts with sizes varying from 423 to 456 bp (Figure $1 \mathrm{~B})$. DNA sequencing of six candidate clones showed that only two had the same sequence. These two clones were chosen for Southern hybridization but did not reveal the initial polymorphism (data not shown). Use of a whole DAF PCR mixture as a probe for hybridization with immobilized candidate clones (3) does not solve the problem if non-polymorphic products with homology to the desired DAF marker band are presented in the mixture. One can expect this if a repeated DNA was

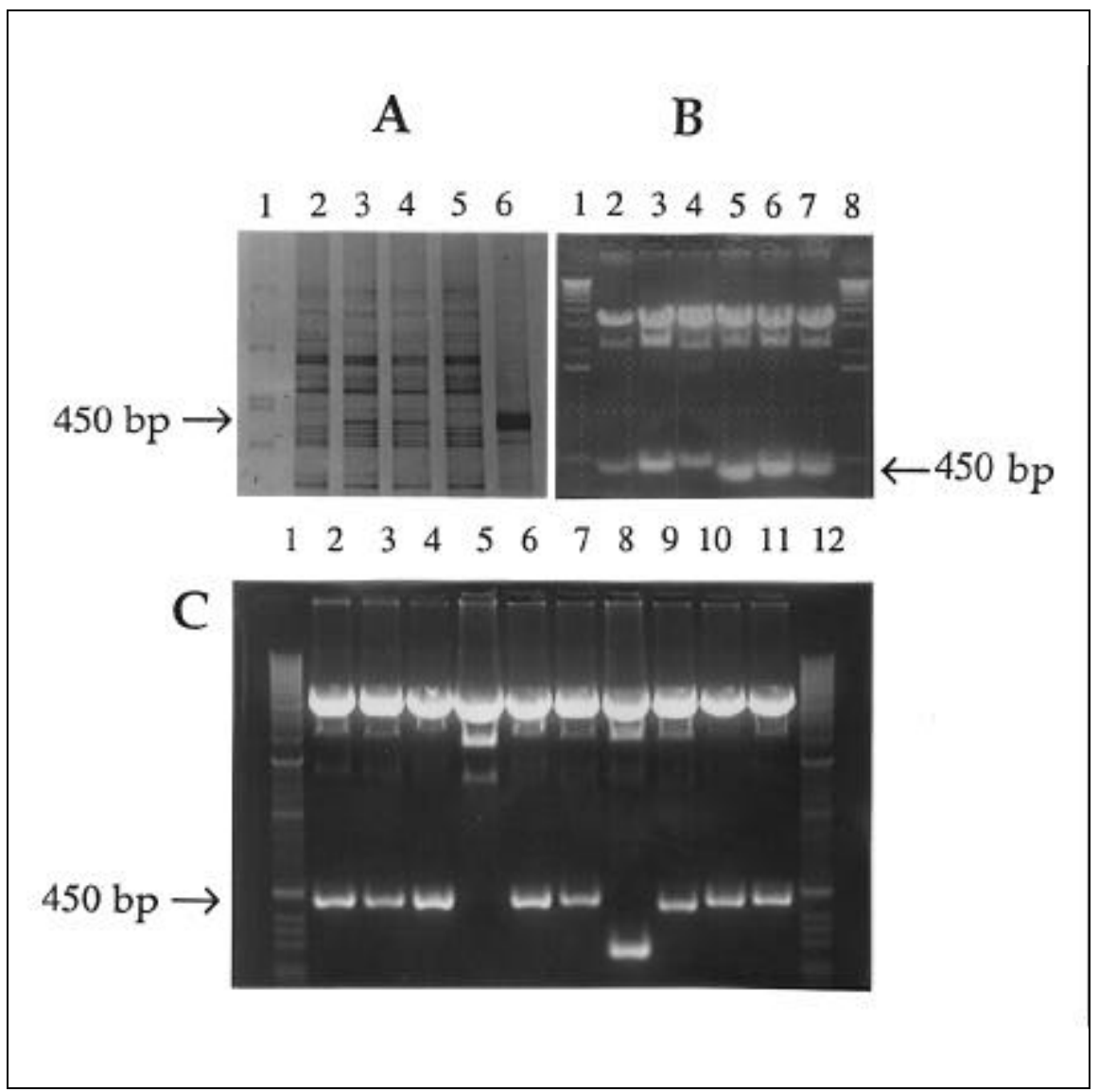

Figure 1. Improved DAF marker cloning. (A) Identification of the 450-bp soybean marker by bulked segregant analysis (4). Lane 1: BioMarker ${ }^{\mathrm{TM}}$ (BioVentures, Murfreesboro, TN, USA); lane 2: wild-type parent; lane 3: mutant parent; lane 4: mutant $\mathrm{F}_{2}$ DNA bulk; lane 5: wild-type bulk; lane 6: re-amplified 450-bp band. (B) $1.5 \%$ agarose gel of EcoRI-digested clones obtained by the standard cloning protocol (6) (lanes 2-7). Lanes 1 and 8: 1-kb DNA ladder (Life Technologies, Gaithersburg, MD, USA). (C) EcoRI-digested clones obtained by the improved protocol (lanes 2-11). Lanes 1 and 12: 1-kb DNA ladder. 
amplified (which is usual for arbitrary PCR methods). Therefore, we optimized the existing cloning protocol (6) as follows.

Once the polymorphism between DAF patterns was confirmed (Figure 1A), the band of interest was excised from the polyacrylamide gel, and the slice was incubated in $20 \mu \mathrm{L}$ TE buffer (10 mM Tris-HCl, $1 \mathrm{mM}$ EDTA, $\mathrm{pH}$ 8.0 ) at $95^{\circ} \mathrm{C}$ for $20 \mathrm{~min}$. Two microliters of the TE eluate [rather than the recommended polyacrylamide gel slice (6)] were used for only one re-amplification with the original DAF primer. The existing protocol required 3-5 rounds of re-amplification (6), which may result in the complex cocktail of re-amplified minor products. Three microliters from the $10-\mu \mathrm{L}$ DAF reamplification mixture were used directly for the cloning in the $\mathrm{pCR}^{\circledR} 2.1 \mathrm{vec}$ tor (Invitrogen, Carlsbad, CA, USA).

We cloned the polymorphic band (Figure 1A) using the improved protocol. Three clones randomly chosen for sequencing (Figure 1C, lanes 2, 3, 6) were identical and confirmed to be the correct ones by differential hybridization with the original polymorphic DAF gel (data not shown). The improved procedure might also be useful for the cloning of polymorphic bands from amplification fragment-length polymorphism (AFLP) (5) and differential display (2) profiles separated by polyacrylamide gels.

\section{REFERENCES}

1.Caetano-Anollés, G., B.J. Bassam and P.M. Gresshoff. 1991. DNA amplification fingerprint using short arbitrary oligonucleotide primers. Bio/Technology 9:553-557.

2.Liang, P. and A.B. Pardee. 1992. Differential display of eucaryotic messenger RNA by means of the polymerase chain reaction. Science 257:967-971.

3.Liu, C. and K.G. Raghothama. 1996. Practical method for cloning cDNAs generated in an mRNA differential display. BioTechniques 20:576-580.

4.Michelmore, R.W., I. Paran and R.V. Kesseli. 1991. Identification of markers linked to disease resistance genes by bulked segregant analysis: a rapid method to detect markers in specific genomic regions using segregating populations. Proc. Natl. Acad. Sci. USA 88:9828-9832.

5.Vos, P., R. Hogers, M. Bleeker, M. Reijans, T. van de Lee, M. Hornes, A. Frijters, J. Pot et al. 1995. AFLP: a new technique for DNA fingerprinting. Nucleic Acids Res. 23:44074414.

6.Weaver, K.R., G. Caetano-Anollés, P.M. Gresshoff and L.M. Callahan. 1994. Isolation and cloning of DNA amplification products from silver-stained polyacrylamide gels. BioTechniques 16:226-227.

We would like to thank the Tennessee Soybean Promotion Board for support and the Molecular Biology Research Facility (UTK) for DNA sequencing. Address correspondence to Peter M. Gresshoff, The University of Tennessee, Plant Molecular Genetics and Center for Legume Research, 269 Plant Science Building, PO Box 1071, Knoxville, TN 37901, USA. Internet: pgresshoff@soybean.ag.utk.edu

Received 7 November 1997; accepted 12 January 1998.

\author{
Artem E. Men and Peter M. \\ Gresshoff \\ The University of Tennessee \\ Knoxville, TN, USA
}

